\title{
BMP signaling components in embryonic transcriptomes of the hover fly Episyrphus balteatus (Syrphidae)
}

Steffen Lemke ${ }^{1,2^{*}}$, Dionysios A Antonopoulos ${ }^{3}$, Folker Meyer ${ }^{3}$, Marc H Domanus ${ }^{3}$ and Urs Schmidt-Ott ${ }^{1^{*}}$

\begin{abstract}
Background: In animals, signaling of Bone Morphogenetic Proteins (BMPs) is essential for dorsoventral (DV) patterning of the embryo, but how BMP signaling evolved with changes in embryonic DV differentiation is largely unclear. Based on the extensive knowledge of BMP signaling in Drosophila melanogaster, the morphological diversity of extraembryonic tissues in different fly species provides a comparative system to address this question. The closest relatives of D. melanogaster with clearly distinct DV differentiation are hover flies (Diptera: Syrphidae). The syrphid Episyrphus balteatus is a commercial bio-agent against aphids and has been established as a model organism for developmental studies and chemical ecology. The dorsal blastoderm of E. balteatus gives rise to two extraembryonic tissues (serosa and amnion), whereas in D. melanogaster, the dorsal blastoderm differentiates into a single extraembryonic epithelium (amnioserosa). Recent studies indicate that several BMP signaling components of D. melanogaster, including the BMP ligand Screw $(\mathrm{Scw})$ and other extracellular regulators, evolved in the dipteran lineage through gene duplication and functional divergence. These findings raise the question of whether the complement of BMP signaling components changed with the origin of the amnioserosa.
\end{abstract}

Results: To search for BMP signaling components in E. balteatus, we generated and analyzed transcriptomes of freshly laid eggs (0-30 minutes) and late blastoderm to early germband extension stages (3-6 hours) using Roche/ 454 sequencing. We identified putative E. balteatus orthologues of $43 \%$ of all annotated D. melanogaster genes, including the genes of all BMP ligands and other BMP signaling components.

Conclusion: The diversification of several BMP signaling components in the dipteran linage of D. melanogaster preceded the origin of the amnioserosa.

[Transcriptome sequence data from this study have been deposited at the NCBI Sequence Read Archive (SRP005289); individually assembled sequences have been deposited at GenBank (JN006969-JN006986).]

\section{Background}

Across animals, the Bone Morphogenetic Protein (BMP) signaling pathway plays a major role in specifying the dorsoventral (DV) axis [1,2]. However, the components of the BMP pathway have been repeatedly modified through lineage specific gene duplications and gene losses $[3,4]$. Whether some of these genetic changes correlate with the origin of species-specific morphological traits that develop under the control of the BMP pathway is unknown. Flies (Diptera) provide an excellent

\footnotetext{
* Correspondence: slemke@uchicago.edu; uschmid@uchicago.edu 'University of Chicago, Dept. of Organismal Biology and Anatomy, CLSC 921B, 920 E. 58th Street, Chicago, IL 60637, USA

Full list of author information is available at the end of the article
}

opportunity to address this question firstly because the BMP signaling pathway of Drosophila melanogaster has been studied in great detail [5,6], and secondly because tissue specification presumably under the control of BMP signaling along the DV axis of dipterans has undergone significant change [7]. In D. melanogaster, dorsal blastoderm differentiates into a single extraembryonic epithelium, called amnioserosa, which closes the developing embryo dorsally [8]. This tissue is found in higher cyclorrhaphan flies (Schizophora), but in other dipterans, dorsal blastoderm gives rise to distinct serosal and amniotic epithelia [9-11]. Serosa and amnion develop from an amnioserosal fold at the margins of the gastrulating embryo. The outer cell layer of this fold

C Biomed Central

(C) 2011 Lemke et al; licensee BioMed Central Ltd. This is an Open Access article distributed under the terms of the Creative Commons Attribution License (http://creativecommons.org/licenses/by/2.0), which permits unrestricted use, distribution, and reproduction in any medium, provided the original work is properly cited. 
becomes the serosa, which closes about the embryo. Its inner cell layer detaches from the serosa but retains continuity with the embryo while closing dorsally (lower cyclorrhaphan flies) or ventrally (non-cyclorrhaphan dipterans). The lower cyclorrhaphan syrphids represent the closest relatives of $D$. melanogaster that have been shown to develop distinct serosa and amnion tissues [9]. Therefore, they are of particular interest in efforts to understand how the origin of the amnioserosa as a new morphology is linked to changes in the underlying developmental gene network.

In previous studies we have characterized the role that the homeobox gene zerknüllt (zen) may have played in the origin of amnioserosa development [reviewed in 7]. The transcription factor Zen is regulated by BMP signaling and essential in serosa specification in non-schizophoran insects and amnioserosa specification in $D$. melanogaster [9,12-14]. In lower Cyclorrhapha and more distant relatives of $D$. melanogaster, zen expression in the serosa is maintained after gastrulation, i. e., when the serosa begins to spread over the embryo [9], whereas in D. melanogaster zen expression in the amnioserosa is down-regulated immediately after gastrulation [13]. In lower cyclorrhaphan flies, postgastrular down-regulation of zen abrogates serosa development and results in the formation of a single extraembryonic tissue with amniotic gene expression [15]. Thus, the repression of this single transcription factor may account for the morphological tissue reorganization that accompanied the origin of the amnioserosa. However, loss of postgastrular zen expression does not explain, why in lower cyclorrhaphan and non-cyclorrhaphan dipterans the patterning of the dorsal blastoderm results in the specification of two distinct extraembryonic tissues types as opposed to one in schizophoran (i. e. higher cyclorrhaphan) flies such as $D$. melanogaster.

In D. melanogaster, amnioserosa specification occurs at the dorsal midline and requires peak-levels of BMP activity $[14,16]$, which are provided through the interaction of two extracellular ligands, Dpp and Scw [17-19]. Both ligands are secreted into the perivitelline space and transported towards the dorsal midline [17,20-24], where BMP-ligand dimers are released from antagonists to activate a receptor complex and initiate intracellular signaling [17,20,25-27]. A cell autonomous autoregulatory loop further increases the BMP signal at the dorsal midline and generates a narrow and sharply delineated domain of BMP peak activity $[20,28]$. Dpp is essential for BMP activity and controls the specification of all tissues that develop under the control of this pathway in the early embryo, including amnioserosa and dorsal ectoderm $[16,29]$. Scw boosts BMP activity along the dorsal midline and is in particular required for amnioserosa specification [19].
In other dipterans, the molecular mechanisms that specify the amnion and serosa are not known. Expression studies in a mosquito suggest that a tighter expression of the Dpp antagonist short gastrulation (Sog) leads to broader BMP signaling, which in turn may allow for the specification of two versus one extraembryonic tissue type [11]. Additionally, Scw is absent from the genomes of mosquitoes and other insects, and it has been suggested that its origin may correlate with the origin of the amnioserosa [3]. Several other BMP signaling components of $D$. melanogaster resulted from gene duplications that have been mapped to the dipteran lineage, while others - known from the BMP pathways of vertebrates - were lost in the lineage leading to $D$. melanogaster [4]. Here we use embryonic transcriptome data of the hover fly Episyrphus balteatus (Syrphidae) to address the question of whether evolutionary changes in the complement of BMP signaling components occurred in correlation with the origin of the amnioserosa. Specifically, we found that with the possible exception of one gene-duplication (crossveinless/shrew) and one gene loss (DAN), genes encoding known BMP signaling components, including $s c w$, are conserved across the schizophoran boundary of the dipteran tree. Thus, most or all of the gains and losses of BMP signaling genes in the dipteran lineage do not correlate with the origin of the amnioserosa, suggesting that the origin of amnioserosa specification was probably achieved by rearranging the interaction of established factors.

\section{Results and Discussion}

\section{Putative Orthologues of 6013 E. balteatus Genes}

We sequenced the transcriptome of $E$. balteatus embryos at two successive time points during early embryogenesis: 0-0.5 hrs old embryos to sample pre-blastoderm stages prior to the onset of zygotic transcription ("maternal library"), and 3-6 hrs old embryos to sample blastoderm and gastrulation stages after the onset of zygotic transcription ("zygotic library"). The cDNA libraries that we prepared from these developmental stages were normalized (Additional file 1A,B) and sequenced using the 454 GS FLX Titanium platform. Following removal of contaminants (see Material and Methods, Additional file 1C, D) reads from both libraries were pooled and assembled using the Newbler Assembler from Roche. Above our chosen cutoff of $100 \mathrm{nt}$, this assembly yielded a total of 16,950 contigs with an average length of $798 \mathrm{nt}(13.5 \mathrm{MB})$ and 26,862 singletons with an average length of $264 \mathrm{nt}$ (7.1 $\mathrm{MB})$. This data set (20.6 MB total sequence data) was used in subsequent analyses (Figure 1A).

To identify E. balteatus genes, we pooled sequence data from both libraries and performed reciprocal BLAST against annotated genes of D. melanogaster. Based on reciprocal hits, we identified putative $D$. melanogaster 

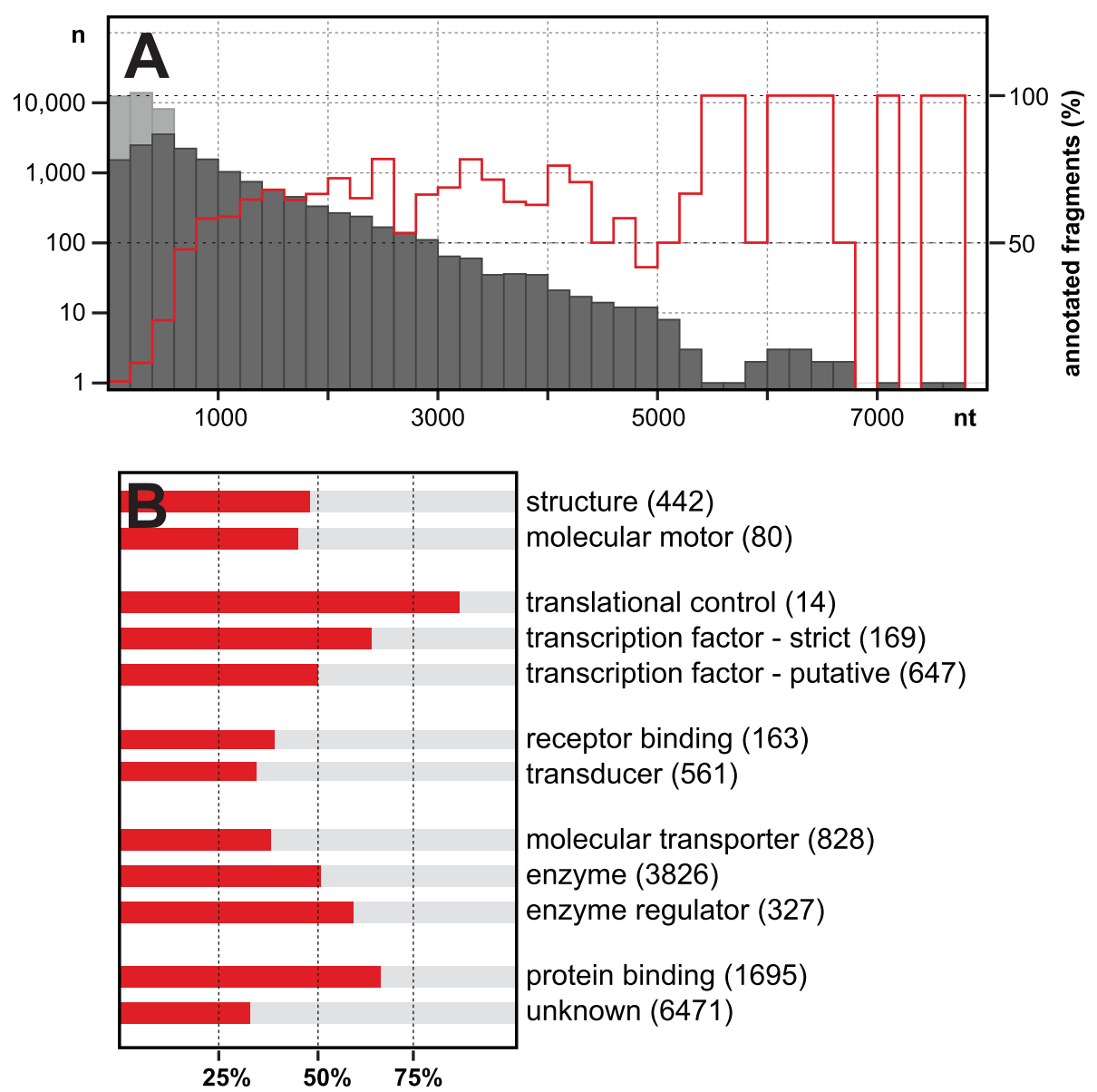

Figure 1 Overview of E. balteatus transcriptome assembly and annotation. (A) Number of sequences per 200-nucleotide-bin ( $y$-axis) is shown as function of assembled sequence lengths (X-axis). Numbers of assembled contigs (dark grey) and singletons (light grey) are shown on a logarithmic scale (left scale), and the proportion of annotated E. balteatus sequences (red graph) are shown in percent (right scale). Note that sequences below $100 \mathrm{nt}$ were not used for further analyses and were excluded from bin 0-200. (B) Gene ontology assignments of annotated $E$. balteatus genes, red bars indicate the proportion of annotated E. balteatus sequences in percent.

orthologues of 6013 E. balteatus genes. In total, about 8.1 MB (39\%) of the assembled E. balteatus sequence data could be annotated (red line in Figure 1A), corresponding to $43 \%$ of annotated D. melanogaster genes. Specifically, we recovered $85 \%$ of genes annotated for translational control, over $60 \%$ of the genes known to encode gene-specific transcription factors (transcription factor - strict [30]), $50 \%$ of the genes associated with known and putative gene-specific transcription factors (transcription factor - putative [30]), about 50\% of genes associated with structural functions (structure) and enzymes, and $30-40 \%$ of genes associated with receptor binding, molecular transporters, and signal transduction (transducer) (Figure 1B).

\section{Assessment of Coverage}

To estimate the coverage of developmental genes, we separately mapped the reads from each library back onto all of 14 previously described E. balteatus segmentation genes, which comprise orthologues of bicoid (Eba-bcd), caudal (Eba-cad), nanos (Eba-nos), torso (Eba-tor), orthodenticle (Eba-otd), hunchback (Eba-hb), Krüppel (EbaKr), knirps (Eba-kni), giant (Eba-gt), hairy (Eba-h), evenskipped (Eba-eve), zerknüllt (Eba-zen), tailless (Eba-tll), and huckebein (Eba-hkb) [9,31-33]. The combined maternal and zygotic coverage of all fourteen genes was on average 8.6-fold (Additional file 2), slightly less than the average coverage of the entire assembled transcriptome ( 12-fold). However, coverage of 5'UTR sequences (2.0fold) and 3'UTR sequences (1.6-fold) was considerably lower than the coverage of ORF sequences (12.5-fold). As the CG content of UTRs (20\%) was notably lower than the CG content of the ORFs (43\%), a systematic bias against $\mathrm{AT}$ rich sequences may have been introduced by less efficient annealing of the random hexamer primers during first strand cDNA synthesis. In any case, coverage 
of our E. balteatus transcriptome data set was high enough to identify at least fragments of genes known to be active during early embryonic development.

All fourteen genes were represented with at least one read from the zygotic library (blue lines in Figure 2A-N), which is consistent with our previous finding that all these genes are expressed in the 3-6 hours time window of embryonic development [9,31-33]. For six of these genes (Eba-bcd, Eba-cad, Eba-nos, Eba-tor, Eba-otd, Eba-hb) we also obtained reads from the maternal library (red lines in Figure 2A-F). Previous and new (Additional file 3A-C) in situ hybridization data indicated maternal expression of Eba-bcd, Eba-cad, Eba-nos, Eba-tor and Eba-otd, but not of Eba-hb. Quantitative PCR (qPCR) on non-normalized cDNA suggested an 18-fold increase of $E b a-h b$ expression levels following the onset of zygotic transcription, whereas expression levels both of Eba-otd and Eba-cad increased by about 2 -fold (orange bars in Additional file 3D). Coverage of these genes in the maternal and zygotic transcriptomes closely reflected our qPCR data (light grey bars in Additional file 3D), suggesting that, despite cDNA normalization, the coverage of these genes remained roughly proportional to their expression levels.

\section{BMP Signaling Components in the E. balteatus Transcriptome Database}

In D. melanogaster, BMP signaling at the dorsal side of the blastoderm is required to specify the amnioserosa as a single extraembryonic tissue (see Background). Based on mosquito data, it has been suggested that in lower dipterans restricted expression of the BMP antagonist Short gastrulation (Sog) may account for an expanded BMP signaling domain in the dorsal blastoderm, which resolves into serosa and amnion territories [11]. Furthermore, it has been suggested that the complement of BMP signaling components changed in the dipteran lineage in parallel with the origin of the amnioserosa [3]. We used our transcriptome database as a tool to test the latter idea by searching for $E$. balteatus homologues of specific BMP signaling components of D. melanogaster.

Specific BMP signaling components include (1) extracellular ligands, (2) transmembrane receptors, (3) intracellular signal transducers, and (4) extracellular modulators of ligands. The D. melanogaster genome contains a total of three genes encoding BMP ligands: decapentaplegic (dpp) [18], glass bottom boat ( $g b b)$ [34], and screw (scw) [19]. These are selectively used depending on the developmental context [5]. In other insects, only homologues of $d p p$ and $g b b$ have been found $[3,4]$. We identified E. balteatus homologues of all three ligands (Figure 3A), indicating that these genes existed prior to the origin of the amnioserosa. Consistent with previous reports $[3,4]$, our gene tree supports a sister gene relationship between $s c w$ and $g b b$. As expected based on a comprehensive survey of TGF- $\beta$ signaling components in the beetle Tribolium castaneum [4], we also identified $E$. balteatus homologues for each of the D. melanogaster TGF- $\beta$ receptors Thickveins (Tkv) and Saxophone (Sax) [35-38], Punt (Put) [39,40], Baboon (Babo) [41], and Wishful thinking (Wit) $[42,43]$, and the SMAD transducers Mothers against Dpp (Mad), Medea, and Smox [44-48], as well as other TGF- $ß$ signaling components (Figure 3B,C; Additional file 4).

In D. melanogaster, activity of BMP ligands is modulated by Sog $[22,23,26]$, which in turn is regulated by the related metalloproteases Tolloid (Tld) and Tolkin (Tok) $[25,49,50]$. We identified E. balteatus homologues of $s o g$ as well as of $t$ ld. While we were not able to identify an orthologue of tok, the presence of a distinct $t$ ld orthologue in $E$. balteatus suggests that the dipteran gene duplication giving rise to $t l d$ and tok occurred before the origin of the amnioserosa (Figure 3D). BMP ligand activity in D. melanogaster is additionally modulated by Twisted gastrulation (Tsg) [51,52], Crossveinless (Cv) [53,54], Shrew (Srw) $[26,55]$, as well as the membrane associated factors Crossveinless-2 (Cv-2) [56,57], Kekkon 5 (Kek5) [58], Pentagone (Pent) [59] and Larval Translucida (Ltl) [60]. We identified E. balteatus homologues of $c v-2$, kek5 (Figure 3E) as well as $t s g, c v$ and an additional $c v$ paralogue, Eba-cv-like (Figure 3F). Previous studies have suggested that $t s g$, $c v$, and $s r w$ originated by two successive duplications of a $c v$-like ancestor in the dipteran lineage [3]. Our gene tree analysis is consistent with this idea, but does not resolve whether Eba-cv-like is orthologous to $s r w$, or whether it is the product of an independent gene duplication in E. balteatus. We did not identify orthologues of pent and $l t l$ in $E$. balteatus, but putative orthologues of both genes are present in the genome of $T$. castaneum (data not shown). Thus, all currently known modulators of BMP ligand activity in D. melanogaster may have existed prior to the origin of the amnioserosa.

Putative orthologues of the vertebrate BMP ligands BMP10 [61] and Anti-Dorsalizing Morphogenetic Protein (ADMP) [62-64], as well as the vertebrate BMP inhibitors BAMBI [65], DAN and Gremlin [66] have been found in beetles and/or wasps but not in D. melanogaster [4]. Among these, we were able to identify a putative orthologue of $D A N$ in E. balteatus (Figure 3G). The loss of this gene may correlate with the origin of the amnioserosa. However, the function of $D A N$ in insects remains unknown and its potential role in BMP signaling is therefore speculative.

\section{Differences in maternal expression of BMP signaling components in $D$. melanogaster and $E$. balteatus}

Based on our finding that coverage levels of segmentation genes in the two sequenced transcriptomes were 

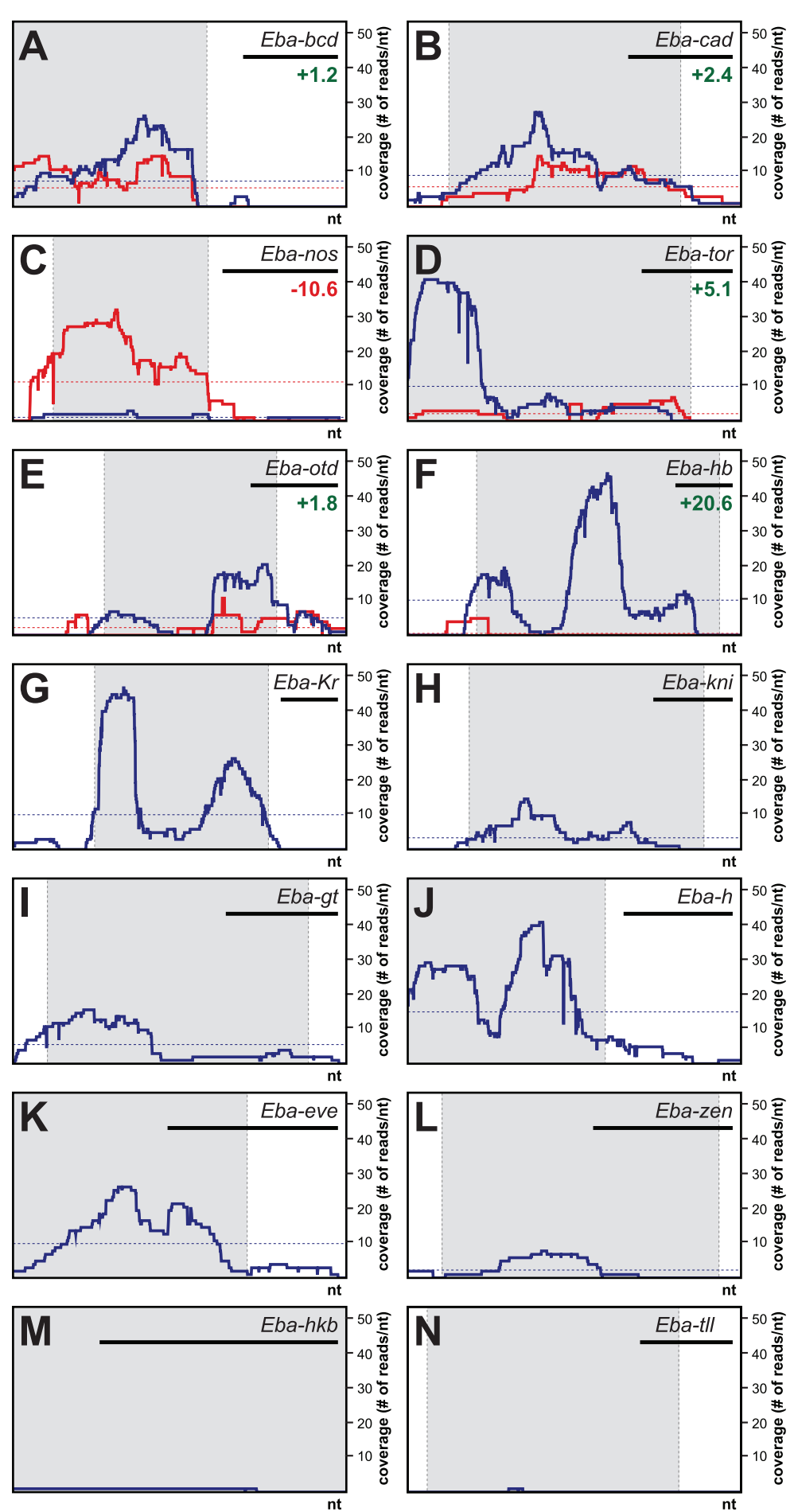

Figure 2 Coverage of previously identified E. balteatus developmental genes. (A) Eba-bcd (GenBank accession: HM044914), (B) Eba-cad (FJ387230), (C) Eba-nos (FJ387226), (D) Eba-tor (HM044920), (E) Eba-otd (FJ387225), (F) Eba-hb (FJ387229), (G) Eba-Kr (HM044918), (H) Eba-kni (HM044916), (I) Eba-gt (HM044915), (J) Eba-h (AY645032), (K) Eba-eve (AY645031), (L) Eba-zen (DQ323932), (M) Eba-kkb (HM067828), and (N) Eba-tll (HM044919). Coverage (i.e., number of reads) is shown as function of nucleotide position in CDNA (X-axis; ORF in grey). Coverage in the maternal cDNA pool (0-0.5 hrs) is labeled in red, coverage in the zygotic cDNA pool (3-6 hrs) is labeled in blue. Average coverage is indicated by dotted horizontal lines. Fold-changes in coverage levels from maternal to zygotic pool are given in green (increase) or red (decrease) numbers. A listing of coverage values detailing ORF and UTR is given in Additional file 2. Scale bars (top right of panel) are $500 \mathrm{nt}$. 
A

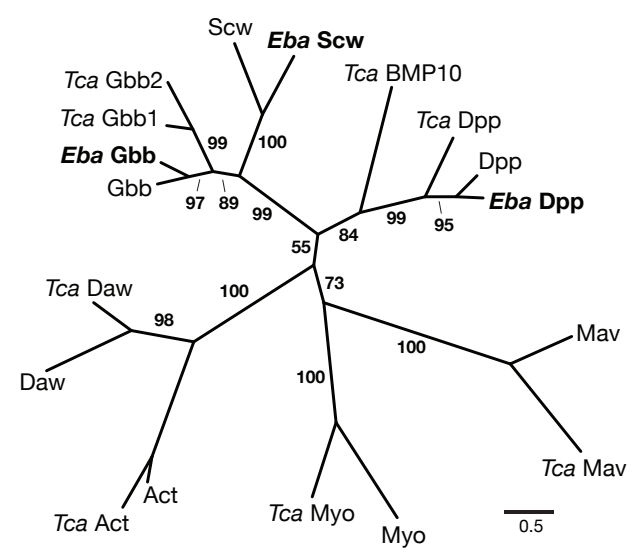

B

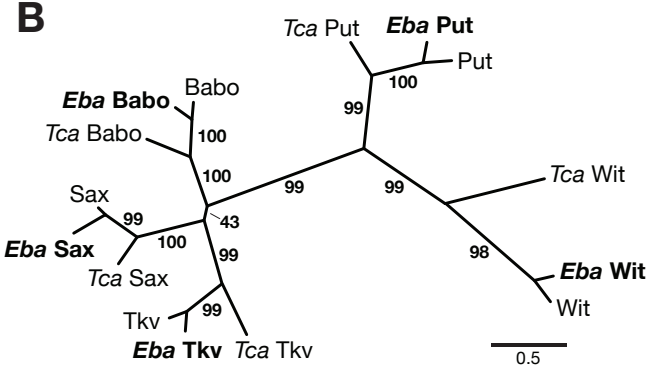

C

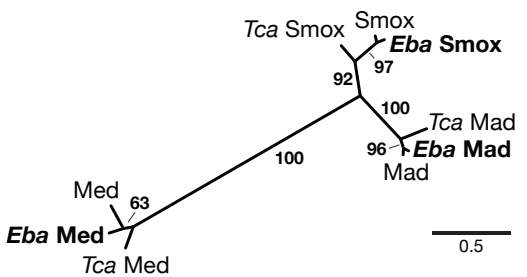

D

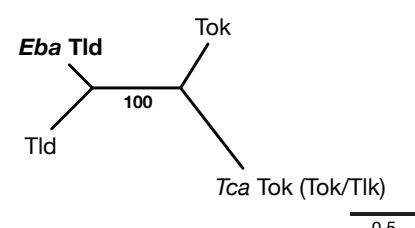

$\mathbf{E}$

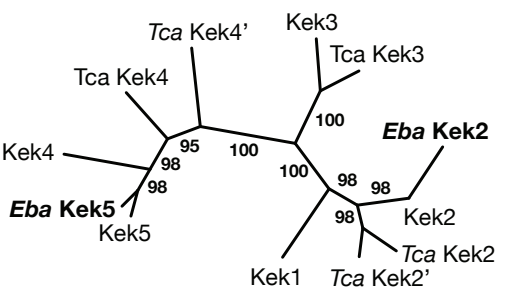

$\mathbf{F}$

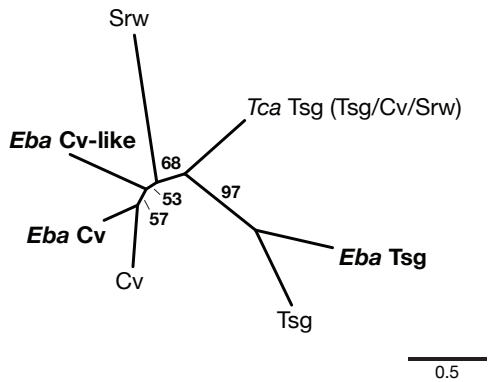

Figure 3 Orthologies of BMP signaling components based on maximum likelihood gene trees using predicted amino acid sequences. (A) TGFß ligands (substitution model DCMut $+\mathrm{i}+\mathrm{g}+\mathrm{g}$ ); (B) TGF $\beta$ type I and type II receptors (substitution model WAG+i+g+f); (C) SMAD family (substitution model $\mathrm{LG}+\mathrm{g}+\mathrm{f}$ ); (D) Metalloproteases related to Tld and Tok (substitution model Dayhoff $+\mathrm{g}+\mathrm{f}$ ); (E) Kekkon family (substitution model $\mathrm{LG}+\mathrm{i}+\mathrm{g}+\mathrm{f}) ;(\mathbf{F})$ Crossveinless family (substitution model Dayhoff $+\mathrm{i}+\mathrm{g}+\mathrm{f}) ;(\mathbf{G})$ DAN family (substitution model Dayhoff $+\mathrm{i}+\mathrm{g}+\mathrm{f}$ ); scale bars indicate estimated changes/position. BeetleBase (T. castaneum) and GenBank accession numbers (all others): (A) Act (Activin- $\beta$; NM_143685), Tca Act (TC015806), Tca BMP10 (TC006506), Daw (dawdle; NM_078737), Tca Daw (TC04297), Dpp (decapentaplegic; NM_057963), Eba Dpp (JN006972), Tca Dpp (TC008466), Gbb (glass bottom boat; NM_057992), Eba Gbb (JN006973), Tca Gbb1 (TC014017), Tca Gbb2 (TC014018), Mav (maverick; NM_079887.2), Tca Mav (TC004299), Myo (myoglianin; NM_166786) Tca Myo (TC015805), Scw (screw; NM_080124.4), Eba Scw (JN006978); (B) Babo (baboon; NM_057652), Eba Babo (JN006969), Tca Babo (TC003240), Put (punt; NM_169591), Eba Put (JN006976), Tca Put (TC011357), Sax (saxophone; NM_078928), Eba Sax (JN006977), Tca Sax (TC015984), Tkv (thickveins; NM_175975), Eba Tkv (JN006980), Tca Tkv (TC006474), Wit (wishful thinking; NM_079953), Eba Wit (JN006986), Tca Wit (TC009314); (C) Mad (mothers against dpp; NM_057669), Eba Mad (JN006974), Tca Mad (TC014924), Med (Medea; NM_079871), Eba Med (JN006975), Tca Med (TC010848), Smox (smad on X; NM_078524), Eba Smox (JN006979), Tca Smox (TC010162); (D) Tld (tolloid; NM_079763), Eba Tld (JN006985), Tok (tolkin; NM_057531), Tca Tok (TC011197); (E) Kek1 (kekkon-1; NM_078835), Kek2 (NM_078827), Tca Kek2 (TC007053), Tca Kek2' (TC008448), Eba Kek2 (JN006983), Kek3 (NM_078851), Tca Kek3 (TC007226), Kek4 (NM_135771), Tca Kek4 (TC007110), Tca Kek4' (TC008070), Kek5 (NM_133154), Eba Kek5 (JN006984); (F) Cv (crossveinless; NM_080525), Eba Cv (JN006982), Eba CV-like (JN006970), Srw (shrew; NM_139629), Tsg (twisted gastrulation; NM_078580), Eba Tsg (JN006981), Tca Tsg (TC003620); (G) Mmu Cerberus (Mus musculus; NM_009887), Mmu Dante (NM_201227), Mmu Gremlin (NM_011824), Tca Gremlin (TC007044), Eba DAN (JN006971), Mmu DAN (NM_008675.2), Tca DAN (TC014861). 
roughly proportional to their expected expression levels (see above; Additional file 3D), we decided to globally compare maternal gene expression between E. balteatus and $D$. melanogaster. For this purpose, we approximated the maternal expression profiles of all annotated E. balteatus genes based on their coverage in the maternal transcriptome and compared them to maternal expression profiles of their D. melanogaster orthologues. Maternal coverage levels of all annotated E. balteatus genes were corrected for sequence lengths and sequencing depth (see Methods) and plotted against coverage levels of their D. melanogaster orthologues, which were estimated from available SOLiD total RNA sequencing data of 0-2 hour old embryos (i.e. stages when the zygotic transcriptome is still essentially silent) [67] (Figure 4). Coverage levels derived from the non-normalized D. melanogaster transcriptome spread by 5.5 orders of magnitude, while those of the normalized E. balteatus transcriptome spread by 3.6 orders of magnitude. A reduced breadth in coverage levels of E. balteatus genes was expected due to the normalization protocol. The coverage levels of the maternal genes nanos, bicoid, torso, and caudal were higher than 1 in the transcriptome of $D$. melanogaster and E. balteatus. In contrast, the zygotic genes huckebein, even skipped, giant, hairy, knirps, tailless, Krüppel, and zerknüllt showed coverage levels lower than 1 in both species (Figure 4A). Notably, the scatter plot correctly revealed the expression differences of hunchback (maternally expressed in D. melanogaster but not in E. balteatus) and orthodenticle (maternally expressed in E. balteatus but not in D. melanogaster). When restricting the data set to BMP components (Figure 4B) or transcription factors (Figure 4C), we readily identified additional candidate maternal expression differences in both gene groups. For example, the data suggest maternal expression of crossveinless- 2 or kekkon5 in E. balteatus but not in D. melanogaster, which might reflect different interactions of BMP signaling molecules and regulators in both species during early embryonic development.

\section{Conclusions}

Comprising orthologous sequences of nearly half (43\%) of all annotated $D$. melanogaster genes, the newly generated transcriptome data of E. balteatus provide a convenient tool to identify putative orthologues of conserved insect genes. Here we used the transcriptome data of $E$. balteatus to show that the novel dipteran BMP ligand Scw and other BMP signaling components of D. melanogaster existed prior to the origin of the amnioserosa (Figure 5). These findings suggest that the origin of amnioserosa development was accompanied by subtle changes in the expression of conserved BMP signaling components, rather than on the origin or loss of individual genes. Modification of the BMP pathway is expected to be constrained due to its multiple functions in development. However, the duplicated genes $(g b b$ and scw; tld and tok; $c v, t s g$ and srw) may have relaxed these constraints, because BMP activity could now be provided by non-identical sets of genes in early (blastoderm) and later developmental stages. We suspect that increasing the role of $s c w$, tld, tsg and $s r w$ in early (blastoderm) development at the expense of $g b b$, tok and $c v$ facilitated genetic accommodation of early DV patterning following the origin of the amnioserosa. Conversely, the entire complement of the duplicated BMP signaling genes might still be required for early DV patterning in lower cyclorrhaphan flies such as E. balteatus.

\section{Methods}

\section{Preparation of Transcriptome Library}

Total RNA was prepared by homogenizing embryos in Trizol (Inivtrogen), treated with DNaseI, and enriched for polyA containing transcripts using the Oligotex kit (Qiagen). First-strand cDNA was synthesized from approximately $1 \mu \mathrm{g}$ of mRNA. Annealing of random hexamer primers $(15 \mathrm{mM})$ was at $25^{\circ} \mathrm{C}$ for 10 minutes, cDNA was synthesized at $50^{\circ} \mathrm{C}$ for 1 hour and followed by inactivation of the reverse transcriptase (Superscript, Invitrogen) at $85^{\circ} \mathrm{C}$ for 5 minutes. Second-strand cDNA was synthesized using the first strand reaction with Klenow DNA Polymerase at $15^{\circ} \mathrm{C}$ for 1.5 hours, and terminated by the addition of $0.5 \mathrm{M}$ EDTA, pH 8. cDNA was purified using the QIAquick MinElute Reaction Cleanup Kit (Qiagen). cDNA ends were filled in ("polished") using a mix of Klenow DNA polymerase and T4 polynucleotide kinase with dNTPs at $20^{\circ} \mathrm{C}$ for 30 minutes, after which the reactions were purified again using the QIAquick MinElute Reaction Clean-up Kit. "A" overhangs were created by incubating polished cDNA with $0.2 \mathrm{mM}$ dATP $0.3 \mathrm{U} / \mu \mathrm{l}$ Klenow exo- at $37^{\circ} \mathrm{C}$ for 30 minutes. cDNA was purified using the QIAquick MinElute Reaction Clean-up Kit (Qiagen), ligated with a mix of AdaptorA and AdaptorB using T4 DNA ligase, and purified again. Adaptors were generated by annealing equimolar amounts of complementary oligos in 2x TNE buffer (20 mM Tris-Cl, pH 8, 0.2 mM EDTA, pH 8, $100 \mathrm{mM}$ $\mathrm{NaCl}$ ). Oligo sequences for both adaptors were adapted from 454 Sequencing Technical Bulletin No. 004-2009, ordered from Integrated DNA Technologies and HPLC purified. Amplification of the library was performed in triplicate using Platinum Taq DNA polymerase HiFi with AdapterA and AdapterB primers. AdapterA FW and AdapterB FW primers. Pooled volumes of the library were purified using the QIAquick MinElute Reaction Clean-up Kit. 

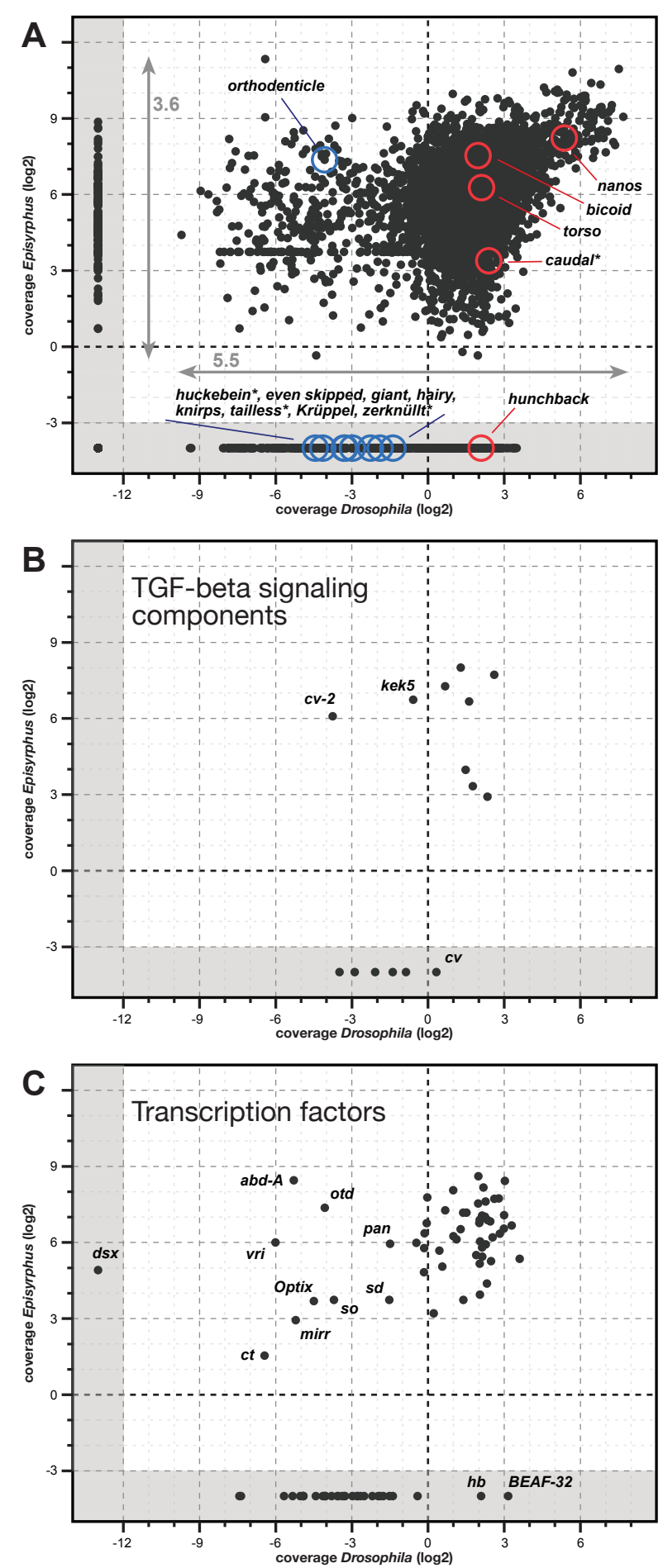

Figure 4 Coverage of maternal E. balteatus genes in comparison with transcriptome data from 0-2 hours old $D$. melanogaster embryos. (A) Scatter plot of orthologous genes for which we determined expression levels in E. balteatus (using 454 data from 0-0.5 hrs old embryos) and D. melanogaster (using RNAseq data from 0-2 hrs old embryos) on a log2 scale. The 14 previously identified E. balteatus genes are circled in red (if their D. melanogaster orthologues are known to have a maternal effect) or in blue (if their D. melanogaster orthologues are thought to lack a maternal effect). Asterisks demark genes that have been manually annotated. Genes not detected at the indicated developmental stage in one of the two species are plotted outside the scale (grey area). (B,C) Same as in (A) but limited to 15 identified genes encoding BMP signaling components (B) or 89 genes of gene specific transcription factors ( $C$, list of genes based on flyTF, see Material and Methods). Genes that might lack significant maternal expression in one of the two species are indicated. Our analysis suggests that the zygotic $D$. melanogaster genes crossveinless2, kekkon-5, doublesex, abdominal-A, vrille and Optix are expressed maternally in E. balteatus, while the maternal D. melanogaster transcription factor BEAF-32 may lack significant maternal expression in E. balteatus. 


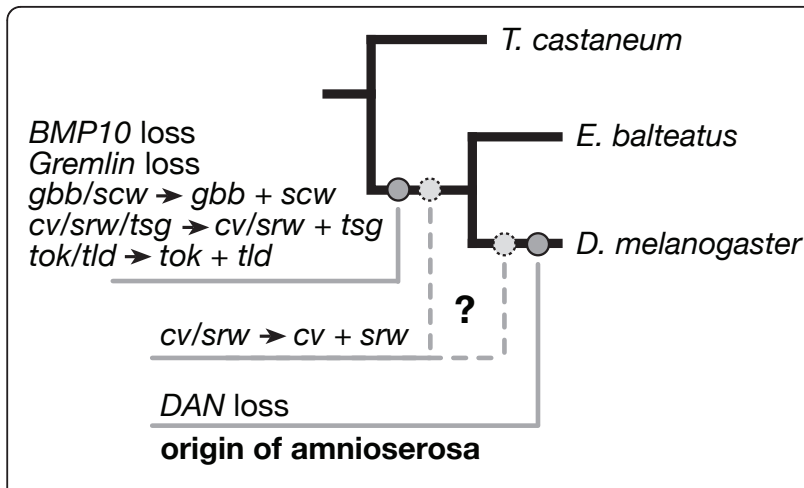

Figure 5 Evolution of BMP signaling components and amnioserosa origin.

\section{Library Normalization and Fragment Size Selection}

Libraries were normalized using the TRIMMER DIRECT cDNA Normalization Kit (Evrogen) and were carried out essentially as described in the user manual. Briefly, $400 \mathrm{ng}$ of each library were suspended in hybridization buffer and split into four tubes. Following $5 \mathrm{hr}$ incubation at $68^{\circ} \mathrm{C}$, the aliquots were treated either with 4 units, 2 units, 1 unit, or no duplex-specific nuclease (DSN). After DSN digestion, the normalized cDNA libraries were amplified by PCR. Optimal amplification within the exponential phase of the PCR was determined visually after electrophoresing different amplification runs of the non-DSN treated sample, after which all aliquots were amplified by a total of 20 cycles. The normalization efficiency was assessed by quantitative PCR (qPCR) of hunchback (Eba-hb, 5'-CTCAGCCCGAATCCAAAT/5'-GGTTGTGGGAGTTGATGTTG, amplicon 137 bp), caudal (Eba-cad, 5'-GAAAGAAT ACTGCACCTCCC/5'-GTCGTTCCGATAGTTGAAGC, amplicon $79 \mathrm{bp}$ ), and alpha-tubulin as reference (Ebatub, 5'-TGAGGCTCGTGAGGATTT/5'-TCACCATCT CCAGAATCCA, amplicon $71 \mathrm{bp})$. Primer efficiency was estimated from a standard curve using five different template concentrations (12.5-200 ng); all analyses were run simultaneously in triplicates. For both libraries, the normalization with 4 units DSN were chosen for sequencing. Based on optimal cycle numbers for amplification and degree of normalization, these libraries were size fractionated using agarose gel electrophoresis, excising fragments at roughly $500 \mathrm{bp}$, and then purifying them using the MinElute Gel Extraction Kit (Qiagen) prior to sequencing.

\section{Sequencing}

Transcriptome library sequencing was performed on the Roche/454 Life Sciences GS-FLX platform at the Institute for Genomics and Systems Biology's (IGSB) HighThroughput Genome Analysis Core (HGAC) at Argonne National Laboratory according to the Roche
GS-FLX XLR70 Titanium emPCR and amplicon sequencing protocols. Each transcriptome library was sequenced on one region of a two region GS-FLX gasket using Roche GS-FLX XLR70 titanium sequencing reagents. An emPCR titration was initially performed on each library to determine the proper bead:library copy ratio that yielded optimal clonal bead percent enrichment to be used in the final bulk XLR70 emPCR reaction.

\section{Sequence Assembly}

Sequencing raw data was processed with gsRunProcessor (software release 2.0.00.20) using standard quality filtering and trimming as defined by the default settings. We obtained 417,735 reads with a mean length of 243 nt for the maternal time point and 406,580 reads with a mean length of $278 \mathrm{nt}$ for the zygotic time point, totaling 214.6 MB of sequence data. This raw data set was contaminated with sequences of the pea aphid Acyrthosiphon pisum, because $E$. balteatus requires aphids for egg deposition, and embryos were collected in batch from leaves heavily infested with aphids. For subsequent analyses, we removed all reads that matched published A. pisum sequences (mRNA + genomic, NCBI, 2009-0713) [68] with $95 \%$ or higher identity. Removal of $A$. pisum sequences reduced the total sequence data by about $26 \%$ to $158.1 \mathrm{MB}$ and the number of reads from each library by about $22 \%$, resulting in 325,200 reads from the maternal library with a mean read length of $228 \mathrm{nt}$, and 311,906 reads from the zygotic library with a mean read length of 269 nt. The distribution of read lengths displayed two peaks, one at about $400 \mathrm{nt}$ (360 $n$ t for reads from the zygotic library) and one at less than $100 \mathrm{nt}$ (Additional file 1C,D). The peak at $400 \mathrm{nt}$ corresponded to the expected mean length using the Titanium chemistry. The peak slightly below 100 nt presumably resulted from our cDNA preparation protocol, which had not yet been optimized for the Titanium chemistry at the time of library preparation and lacked, for example, any additional size exclusion steps following gel electrophoreses.

All reads have been deposited as the E. balteatus transcriptome at the NCBI Sequence Read Archive (SRA, SRP005289). Assembly was based on combined SFF sequence files of the maternal and zygotic libraries using Newbler Assembler (software release 2.3). Newbler parameters were default except: minimal identity of $90 \%$ in overlaps (-mi 90), overlaps to be at least $30 \mathrm{nt}$ in length $(-\mathrm{ml} 30)$, minimal length of contigs to be $100 \mathrm{nt}(-1$ 100). Newbler was run as cDNA assembly (-cdna), which includes processing of contigs that are found to be variants of the same transcript into distinct isotigs. From a total of 637,080 reads, 544,776 reads (85.5\%) were assembled (fully assembled reads: 432,160 reads, 
67.8\%; partially assembled reads: 112,616 reads, $17.7 \%$ ). The remaining reads were singletons $(56,625$ reads; $8.9 \%)$ or excluded as either originating from repeat regions (221 reads; $0.03 \%)$, outliers (23,060 reads; $3.6 \%)$, or too short (< 50 base pairs: 12,398 reads; $1.9 \%$ ) (see Additional file 4 for comparison with Newbler 2.5.3).

\section{Sequence Annotation}

Reported isotigs $(12,296 ; 12.9 \mathrm{MB})$ and singletons of at least $100 \mathrm{nt}$ length $(26,862 ; 7.1 \mathrm{MB}$; identified from 454ReadStatus.txt) were combined, and reciprocal BLAST searches of the E. balteatus transcriptome were carried out against the translated transcriptome of $D$. melanogaster (dmel-all-translation-r5.29.faa, flybase.org) using blastx ( $E$. balteatus query against $D$. melanogaster database) and tblastn (D. melanogaster query against $E$. balteatus database). Annotation was performed with an e-value threshold of 10 to screen for all putative orthologues ("no cutoff") and with an e-value threshold of 1e10 to obtain a conservative list of high confidence orthologues ("1e-10") (see Additional file 5 for comparison of annotation based on assemblies with Newbler 2.3 and Newbler 2.5.3). Reciprocal hits of E. balteatus with $D$. melanogaster were assigned with the same flybase $D$. melanogaster CG identifiers. Gene ontology terms were assigned based on the current $D$. melanogaster gene ontology, with the exception of 'transcription factor strict' and 'transcription factor - putative', which were based on a curated list of genes of known and putative gene specific transcription factors [30].

\section{Sequence Coverage}

To determine coverage levels for individual published $E$. balteatus genes, associated reads of the maternal and zygotic library sequences were identified by BLAST search (blastn) and assembled with the published sequence using CAP3 with standard parameters [69]. Coverage levels were then calculated for each nucleotide position of the published gene sequences. Fold-coverage in the maternal library was used to approximate maternal expression levels of all annotated E. balteatus genes. The assembled E. balteatus transcriptome (isotigs and singletons) was blasted against all maternal reads (blastn). Coverage of annotated transcripts was then calculated from all reads that matched with at least 95\% identity and over the length of at least $50 \mathrm{nt}$. To approximate levels of gene expression, coverage of each gene was divided by its sequence length and the total RNAseq data of the maternal library $(74 \mathrm{MB})$. Fold-coverage in the 0-2 hr time point of the D. melanogaster development transcriptome was used to approximate maternal expression levels of $D$. melanogaster genes [67]. Expression data of $D$. melanogaster genes in $0-2$ hrs old embryos was downloaded from modEncode as coverage data mapped onto the entire genome (BC1_plus.wig, BC1_minus.wig). Expression of gene transcripts was retrieved from this genomic map by extracting coverage information of all exons for each annotated gene (BDGP/ dm3). To account for potentially mis-annotated exonintron structures of computationally predicted genes, gene expression was approximated by the coverage of the most strongly expressed exon longer than $500 \mathrm{nt}$, or by the coverage of the most strongly expressed transcript variant, whichever was higher. To approximate levels of gene expression, coverage of each gene was divided by its sequence length and the total RNAseq data of the 0-2 hr time point (3.4 GB, SRP001696).

\section{Gene Discovery of BMP Signaling Components}

A list of D. melanogaster and Tribolium castaneum BMP signaling compounds was matched against assembled and unassembled E. balteatus transcriptome data (tblastn, e-value of 0.001). Identified E. balteatus sequences were assembled in Sequencher, assemblies were manually corrected for ORF frame shifts by alignment with D. melanogaster protein sequence, and sequence orthology was confirmed by reciprocal blast against a $D$. melanogaster database. To exhaustively search for putative duplicates specific to the E. balteatus lineage, blast searches were repeated using lower cut-offs (increasingly higher e-values) until all newly identified and assembled E. balteatus reads matched a clearly nonorthologous sequence in available insect protein databases. All T. castaneum sequences were retrieved from BeetleBase (ftp://bioinformatics.ksu.edu/pub/BeetleBase/ latest/Sequences/Tribolium_Official_Gene_Sequences/ mRNA.fa).

\section{Phylogenetic Gene Trees}

Protein alignments were created using the Clustal algorithm with standard parameters (MegAlign). When more than half of the aligned sequences carried a gap at a given position, these positions were removed from the alignment. The amino acid substitution model was estimated using AIC in ProtTest [70]; maximum likelihood trees were calculated using PhyML [71]. Bootstrap values were based on 1000 replicas. Trees were plotted with drawtree (Phylip package) [72] and the newick-utils package [73].

Custom scripts (Perl, R) were used to automate blast searches and evaluation, calculate E. balteatus and D. melanogaster gene coverage, and prune sequence alignments. Scripts are available on request. Plots were prepared with gnuplot and finished with Freehand. Assembly, blast searches, and bootstrap analysis were computed on the computer cluster of the Department of Ecology \& Evolution at the University of Chicago (http://biocomputing.uchicago. edu). 


\section{Additional material}

\section{Additional file 1: Normalization and 454 sequencing of} transcriptome libraries. (A) Normalization of library of 0-0.5 hrs old embryos. (B) Normalization of library of 3-6 hrs old embryos. Normalization was assessed by quantitative PCR. Shown are transcript levels of E. balteatus alpha tubulin (Eba-tub) relative to E. balteatus orthologues of hunchback (Eba-hb) and caudal (Eba-Cad). Bar heights indicate transcript levels, colors indicate levels in the non-normalized CDNA library (blue), and after normalization with 1 unit DSN (red), 2 units DSN (orange), and 4 units DSN (yellow). (C) Distribution of read lengths of library from 0-0.5 hrs old embryos. (D) Distribution of read lengths of library from 3-6 hrs old embryos. Read length (x-axis) is shown as a function of number of reads ( $y$-axis) before (grey graph) and after removal of pea aphid sequence contaminations (black graph).

Additional file 2: Coverage of previously identified $E$. balteatus embryonic patterning genes in the libraries of 0-0.5 hrs old embryos (maternal) and 3-6 hrs old embryos (zygotic).

Additional file 3: Expression of Eba-otd in ovarian follicles and early embryos. (A-C) Eba-otd in situ hybridizations of ovarian follicles $(A, B)$ and an early embryo (C). Anterior is left, dorsal is up. (D) Increase of zygotic expression levels relative to maternal expression levels measured for Ebaotd, Eba-cad, and Eba-hb using qPCR (orange bars) and transcriptome coverage (grey bars). qPCR data are based on the average of two independent PCRs, each of which run in triplicate. Bars indicate variance between samples.

Additional file 4: Schematic overview of TGF- $\beta$ signaling in $D$. melanogaster. TGF- $\beta$ ligands bind as dimers to a transmembrane receptor complex, which activates signal transducing protein that translocates to the nucleus to regulate gene activity (modified after [74]). Components of the BMP signaling cascade are shown in red, components of the Activin- $\beta$ signaling cascade in blue, shared components in purple. In the case of generalized BMP signaling, extraecellular ligands (Dpp, Scw, Gbb) are regulated by BMP antagonists Sog and Tsg/Cv/Srw(?), and metalloprotease activity of Tld/Tok, which cleaves Sog and frees BMP. Additional BMP specific regulators are transmembrane protein $\mathrm{CV}-2$, which directly binds BMPs, and membrane associated protein Kek5. Active BMPs signal through the receptor complex composed of Tkv, Sax and Put, which phophorylates Mad. Phophorylated Mad recruits Medea and translocates to the nucleus. For abbreviations, see legend to Figure 3.

Additional file 5: Comparison of sequence annotation following de novo transcriptome assemblies produced by Newbler v2.3 and Newbler v2.5.3. During manuscript preparation, a new version of Newbler became available (v2.5). To address the concern of a potential underperformance of Newbler v2.3 in the case of our data set, we repeated the assembly and annotation with the latest available Newbler assembler (v2.5.3) and compared the results to the assembly with Newbler v2.3. We found the total number of assembled bases with Newbler v2.5.3 (10.5 Mb) decreased by almost 20\% when compared with the number of assembled bases with Newbler v2.3 (12.9 Mb), suggesting that for our dataset Newbler v2.5.3 performed with more stringent assembly conditions. The observed differences did not affect our identification of BMP signaling components in E. balteatus as these genes were all identified by Blast and subsequent manual assembly.

\section{Acknowledgements}

We thank Lucien Jarymowycz, IT systems manager in the Department of Organismal Biology at the University of Chicago, for his professional help. Funding was provided by NSF grants 0719445 and 0840687 to U. S.-O.

\section{Author details}

${ }^{1}$ University of Chicago, Dept. of Organismal Biology and Anatomy, CLSC 921B, 920 E. 58th Street, Chicago, IL 60637, USA. ${ }^{2}$ Current Address: University of Heidelberg, Centre for Organismal Studies, Im Neuenheimer Feld 230, 69120 Heidelberg, Germany. ${ }^{3}$ Argonne National Laboratory, Institute for Genomics \& Systems Biology, 9700 S. Cass Avenue, Argonne, IL 60439, USA.

\section{Authors' contributions}

$\mathrm{SL}$ designed the research, performed the bioinformatic analyses and wrote the manuscript. DAA prepared the cDNA libraries for 454 sequencing, FM and MHD performed 454 sequencing. USO designed the research, analyzed the data, wrote the manuscript and obtained funding. All authors read and approved the final manuscript.

Received: 28 January 2011 Accepted: 31 May 2011

Published: 31 May 2011

\section{References}

1. Niehrs C: On growth and form: a Cartesian coordinate system of Wnt and BMP signaling specifies bilaterian body axes. Development 2010, 137(6):845-857.

2. De Robertis EM: Spemann's organizer and the self-regulation of embryonic fields. Mech Dev 2009, 126(11-12):925-941.

3. Fritsch C, Lanfear R, Ray RP: Rapid evolution of a novel signalling mechanism by concerted duplication and divergence of a BMP ligand and its extracellular modulators. Dev Genes Evol 2010, 220(910):235-250.

4. Van der Zee M, da Fonseca RN, Roth S: TGFbeta signaling in Tribolium: vertebrate-like components in a beetle. Dev Genes Evol 2008, 218(34):203-213.

5. O'Connor MB, Umulis D, Othmer HG, Blair SS: Shaping BMP morphogen gradients in the Drosophila embryo and pupal wing. Development 2006, 133(2):183-193.

6. Umulis D, O'Connor MB, Blair SS: The extracellular regulation of bone morphogenetic protein signaling. Development 2009, 136(22):3715-3728.

7. Schmidt-Ott U, Rafiqi AM, Lemke S: Hox3/zen and the Evolution of Extraembryonic Epithelia in Insects. In Hox Genes' Studies from the 20th to the 21st Century. Edited by: Deutsch J. Austin, TX: Landes Biosciences; 2010:

8. Campos-Ortega J, Hartenstein V: The embryonic development of Drosophila melanogaster. Berlin, Heidelberg, New York: Springer Verlag; 2 1997.

9. Rafiqi AM, Lemke S, Ferguson S, Stauber M, Schmidt-Ott U: Evolutionary origin of the amnioserosa in cyclorrhaphan flies correlates with spatial and temporal expression changes of zen. Proc Natl Acad Sci USA 2008, 105(1):234-239.

10. Goltsev Y, Rezende G, Vranizan K, Lanzaro G, Valle D, Levine M: Developmental and evolutionary basis for drought tolerance of the Anopheles gambiae embryo. Dev Biol 2009, 330:462-470.

11. Goltsev Y, Fuse N, Frasch M, Zinzen RP, Lanzaro G, Levine M: Evolution of the dorsal-ventral patterning network in the mosquito, Anopheles gambiae. Development 2007, 134(13):2415-2424.

12. van der Zee M, Berns N, Roth S: Distinct functions of the Tribolium zerknüllt genes in serosa specification and dorsal closure. Curr Biol 2005, 15:624-636.

13. Rushlow $C$, Levine $M$ : Role of the zerknüllt gene in dorsal-ventral pattern formation in Drosophila. Adv Genet 1990, 27:277-307.

14. Ray RP, Arora K, Nüsslein-Volhard C, Gelbart WM: The control of cell fate along the dorsal-ventral axis of the Drosophila embryo. Development 1991, 113(1):35-54.

15. Rafiqi AM, Lemke S, Schmidt-Ott U: Postgastrular zen expression is required to develop distinct amniotic and serosal epithelia in the scuttle fly Megaselia. Dev Biol 2010, 341(1):282-90.

16. Ferguson EL, Anderson KV: Decapentaplegic acts as a morphogen to organize dorsal-ventral pattern in the Drosophila embryo. Cell 1992, 71(3):451-461.

17. Shimmi $\mathrm{O}$, Umulis $\mathrm{D}$, Othmer $\mathrm{H}, \mathrm{O}^{\prime}$ Connor MB: Facilitated transport of a $\mathrm{Dpp} / \mathrm{Scw}$ heterodimer by Sog/Tsg leads to robust patterning of the Drosophila blastoderm embryo. Cell 2005, 120(6):873-886.

18. Padgett RW, St Johnston RD, Gelbart WM: A transcript from a Drosophila pattern gene predicts a protein homologous to the transforming growth factor-beta family. Nature 1987, 325(6099):81-84.

19. Arora K, Levine MS, O'Connor MB: The screw gene encodes a ubiquitously expressed member of the TGF-beta family required for specification of dorsal cell fates in the Drosophila embryo. Genes Dev 1994, 8(21):2588-2601.

20. Wang YC, Ferguson EL: Spatial bistability of Dpp-receptor interactions during Drosophila dorsal-ventral patterning. Nature 2005, 434(7030):229-234 
21. Eldar A, Dorfman R, Weiss D, Ashe H, Shilo BZ, Barkai N: Robustness of the BMP morphogen gradient in Drosophila embryonic patterning. Nature 2002, 419(6904):304-308

22. Decotto $E$, Ferguson EL: A positive role for Short gastrulation in modulating BMP signaling during dorsoventral patterning in the Drosophila embryo. Development 2001, 128(19):3831-3841.

23. Ashe HL, Levine M: Local inhibition and long-range enhancement of Dpp signal transduction by Sog. Nature 1999, 398(6726):427-431.

24. Francois V, Solloway M, O'Neill JW, Emery J, Bier E: Dorsal-ventral patterning of the Drosophila embryo depends on a putative negative growth factor encoded by the short gastrulation gene. Genes Dev 1994, 8(21):2602-2616

25. Marqués G, Musacchio M, Shimell MJ, Wünnenberg-Stapleton K, Cho KW, O'Connor MB: Production of a DPP activity gradient in the early Drosophila embryo through the opposing actions of the SOG and TLD proteins. Cell 1997, 91(3):417-426.

26. Ferguson EL, Anderson KV: Localized enhancement and repression of the activity of the TGF-beta family member, decapentaplegic, is necessary for dorsal-ventral pattern formation in the Drosophila embryo. Development 1992, 114(3):583-597.

27. Shimell MJ, Ferguson EL, Childs SR, O'Connor MB: The Drosophila dorsalventral patterning gene tolloid is related to human bone morphogenetic protein 1. Cell 1991, 67(3):469-481.

28. Umulis DM, Serpe M, O'Connor MB, Othmer HG: Robust, bistable patterning of the dorsal surface of the Drosophila embryo. Proc Natl Acad Sci USA 2006, 103(31):11613-11618.

29. Wharton KA, Ray RP, Gelbart WM: An activity gradient of decapentaplegic is necessary for the specification of dorsal pattern elements in the Drosophila embryo. Development 1993, 117(2):807-822.

30. Pfreundt U, James DP, Tweedie S, Wilson D, Teichmann SA, Adryan B: FlyTF: improved annotation and enhanced functionality of the Drosophila transcription factor database. Nucleic Acids Res 2010, 38:443-447.

31. Lemke S, Busch S, Antonopoulos D, Meyer F, Domanus M, Schmidt-Ott U: Maternal activation of gap genes in the hover fly Episyrphus. Development 2010, 137(10):1709-1719

32. Lemke S, Schmidt-Ott U: Evidence for a composite anterior determinant in the hover fly Episyrphus balteatus (Syrphidae), a cyclorrhaphan fly with an anterodorsal serosa anlage. Development 2009, 136:117-127.

33. Bullock SL, Stauber M, Prell A, Hughes JR, Ish-Horowicz D, Schmidt-Ott U: Differential cytoplasmic mRNA localisation adjusts pair-rule transcription factor activity to cytoarchitecture in dipteran evolution. Development 2004, 131(17):4251-4261.

34. Doctor JS, Jackson PD, Rashka KE, Visalli M, Hoffmann FM: Sequence, biochemical characterization, and developmental expression of a new member of the TGF-beta superfamily in Drosophila melanogaster. Dev Biol 1992, 151(2):491-505.

35. Xie T, Finelli AL, Padgett RW: The Drosophila saxophone gene: a serinethreonine kinase receptor of the TGF-beta superfamily. Science 1994, 263(5154):1756-1759.

36. Brummel TJ, Twombly V, Marqués G, Wrana JL, Newfeld SJ, Attisano L, Massagué J, O'Connor MB, Gelbart WM: Characterization and relationship of Dpp receptors encoded by the saxophone and thick veins genes in Drosophila. Cell 1994, 78(2):251-261.

37. Nellen D, Affolter M, Basler K: Receptor serine/threonine kinases implicated in the control of Drosophila body pattern by decapentaplegic. Cell 1994, 78(2):225-237.

38. Penton A, Chen Y, Staehling-Hampton K, Wrana JL, Attisano L, Szidonya J, Cassill JA, Massagué J, Hoffmann FM: Identification of two bone morphogenetic protein type I receptors in Drosophila and evidence that Brk25D is a decapentaplegic receptor. Cell 1994, 78(2):239-250.

39. Letsou A, Arora K, Wrana JL, Simin K, Twombly V, Jamal J, StaehlingHampton K, Hoffmann FM, Gelbart WM, Massagué J: Drosophila Dpp signaling is mediated by the punt gene product: a dual ligand-binding type II receptor of the TGF beta receptor family. Cell 1995, 80(6):899-908.

40. Ruberte E, Marty T, Nellen D, Affolter M, Basler K: An absolute requirement for both the type II and type I receptors, punt and thick veins, for $d p p$ signaling in vivo. Cell 1995, 80(6):889-897

41. Brummel T, Abdollah S, Haerry TE, Shimell MJ, Merriam J, Raftery L, Wrana JL, O'Connor MB: The Drosophila activin receptor baboon signals through dSmad2 and controls cell proliferation but not patterning during larval development. Genes Dev 1999, 13(1):98-111.

42. Aberle H, Haghighi AP, Fetter RD, McCabe BD, Magalhães TR, Goodman CS: wishful thinking encodes a BMP type II receptor that regulates synaptic growth in Drosophila. Neuron 2002, 33(4):545-558.

43. Marqués G, Bao H, Haerry TE, Shimell MJ, Duchek P, Zhang B, O'Connor MB: The Drosophila BMP type II receptor Wishful Thinking regulates neuromuscular synapse morphology and function. Neuron 2002, 33(4):529-543.

44. Sekelsky JJ, Newfeld SJ, Raftery LA, Chartoff EH, Gelbart WM: Genetic characterization and cloning of mothers against dpp, a gene required for decapentaplegic function in Drosophila melanogaster. Genetics 1995, 139(3):1347-1358.

45. Wisotzkey RG, Mehra A, Sutherland DJ, Dobens LL, Liu X, Dohrmann C, Attisano L, Raftery LA: Medea is a Drosophila Smad4 homolog that is differentially required to potentiate DPP responses. Development 1998, 125(8):1433-1445.

46. Hudson JB, Podos SD, Keith K, Simpson SL, Ferguson EL: The Drosophila Medea gene is required downstream of $d p p$ and encodes a functional homolog of human Smad4. Development 1998, 125(8):1407-1420.

47. Das P, Maduzia LL, Wang H, Finelli AL, Cho SH, Smith MM, Padgett RW: The Drosophila gene Medea demonstrates the requirement for different classes of Smads in dpp signaling. Development 1998, 125(8):1519-1528.

48. Henderson KD, Andrew DJ: Identification of a novel Drosophila SMAD on the X chromosome. Biochem Biophys Res Commun 1998, 252(1):195-201.

49. Srinivasan S, Rashka KE, Bier E: Creation of a Sog morphogen gradient in the Drosophila embryo. Dev Cell 2002, 2(1):91-101.

50. Serpe M, Ralston A, Blair SS, O'Connor MB: Matching catalytic activity to developmental function: tolloid-related processes Sog in order to help specify the posterior crossvein in the Drosophila wing. Development 2005, 132(11):2645-2656

51. Mason ED, Konrad KD, Webb CD, Marsh JL: Dorsal midline fate in Drosophila embryos requires twisted gastrulation, a gene encoding a secreted protein related to human connective tissue growth factor. Genes Dev 1994, 8(13):1489-1501.

52. Ross JJ, Shimmi O, Vilmos P, Petryk A, Kim H, Gaudenz K, Hermanson S, Ekker SC, O'Connor MB, Marsh JL: Twisted gastrulation is a conserved extracellular BMP antagonist. Nature 2001, 410(6827):479-483.

53. Shimmi O, Ralston A, Blair SS, O'Connor MB: The crossveinless gene encodes a new member of the Twisted gastrulation family of BMPbinding proteins which, with Short gastrulation, promotes BMP signaling in the crossveins of the Drosophila wing. Dev Biol 2005, 282(1):70-83.

54. Vilmos $P$, Sousa-Neves $R$, Lukacsovich $T$, Marsh JL: crossveinless defines a new family of Twisted-gastrulation-like modulators of bone morphogenetic protein signalling. EMBO Rep 2005, 6(3):262-267.

55. Bonds M, Sands J, Poulson W, Harvey C, Von Ohlen T: Genetic screen for regulators of ind expression identifies shrew as encoding a novel twisted gastrulation-like protein involved in Dpp signaling. Dev Dyn 2007, 236(12):3524-3531.

56. Serpe M, Umulis D, Ralston A, Chen J, Olson DJ, Avanesov A, Othmer $H_{\text {, }}$ O'Connor MB, Blair SS: The BMP-binding protein Crossveinless 2 is a short-range, concentration-dependent, biphasic modulator of BMP signaling in Drosophila. Dev Cell 2008, 14(6):940-953.

57. Conley CA, Silburn R, Singer MA, Ralston A, Rohwer-Nutter D, Olson DJ, Gelbart W, Blair SS: Crossveinless 2 contains cysteine-rich domains and is required for high levels of BMP-like activity during the formation of the cross veins in Drosophila. Development 2000, 127(18):3947-3959.

58. Evans TA, Haridas H, Duffy JB: Kekkon5 is an extracellular regulator of BMP signaling. Dev Biol 2009, 326(1):36-46.

59. Vuilleumier R, Springhorn A, Patterson L, Koidl S, Hammerschmidt M, Affolter M, Pyrowolakis G: Control of Dpp morphogen signalling by a secreted feedback regulator. Nat Cell Biol 2010, 12(6):611-617.

60. Szuperák M, Salah S, Meyer EJ, Nagarajan U, Ikmi A, Gibson MC: Feedback regulation of Drosophila BMP signaling by the novel extracellular protein Larval Translucida. Development 2011, 138(4):715-724.

61. Chen H, Shi S, Acosta L, Li W, Lu J, Bao S, Chen Z, Yang Z, Schneider MD, Chien KR, et al: BMP10 is essential for maintaining cardiac growth during murine cardiogenesis. Development 2004, 131(9):2219-2231. 
62. Moos M, Wang S, Krinks M: Anti-dorsalizing morphogenetic protein is a novel TGF-beta homolog expressed in the Spemann organizer. Development 1995, 121(12):4293-4301.

63. Joubin K, Stern CD: Molecular interactions continuously define the organizer during the cell movements of gastrulation. Cell 1999, 98(5):559-571.

64. Lele Z, Nowak M, Hammerschmidt M: Zebrafish admp is required to restrict the size of the organizer and to promote posterior and ventral development. Dev Dyn 2001, 222(4):681-687.

65. Onichtchouk D, Chen YG, Dosch R, Gawantka V, Delius H, Massagué J, Niehrs C: Silencing of TGF-beta signalling by the pseudoreceptor BAMBI. Nature 1999, 401(6752):480-485.

66. Hsu DR, Economides AN, Wang X, Eimon PM, Harland RM: The Xenopus dorsalizing factor Gremlin identifies a novel family of secreted proteins that antagonize BMP activities. Mol Cell 1998, 1(5):673-683.

67. Graveley BR, Brooks AN, Carlson JW, Duff MO, Landolin JM, Yang L, Artieri CG, van Baren MJ, Boley N, Booth BW, et al: The developmental transcriptome of Drosophila melanogaster. Nature 2010.

68. Consortium TIAG: Genome sequence of the pea aphid Acyrthosiphon pisum. PLoS Biol 2010, 8(2):e1000313.

69. Huang X, Madan A: CAP3: A DNA sequence assembly program. Genome Res 1999, 9:868-877.

70. Abascal F, Zardoya R, Posada D: ProtTest: selection of best-fit models of protein evolution. Bioinformatics 2005, 21:2104-2105.

71. Guindon S, Gascuel O: A simple, fast, and accurate algorithm to estimate large phylogenies by maximum likelihood. Syst Biol 2003, 52(5):696-704.

72. Felsenstein J: PHYLIP (Phylogeny Inference Package) version 3.6. Distributed by the author. Department of Genome Sciences, University of Washington, Seattle 2005.

73. Junier T, Zdobnov EM: The Newick utilities: high-throughput phylogenetic tree processing in the Unix shell. Bioinformatics 2010.

74. Van der Zee M, da Fonseca RN, Roth S: TGFbeta signaling in Tribolium: vertebrate-like components in a beetle. Dev Genes Evol 2008, 218(34):203-213.

doi:10.1186/1471-2164-12-278

Cite this article as: Lemke et al.: BMP signaling components in embryonic transcriptomes of the hover fly Episyrphus balteatus (Syrphidae). BMC Genomics 2011 12:278.

\section{Submit your next manuscript to BioMed Central and take full advantage of:}

- Convenient online submission

- Thorough peer review

- No space constraints or color figure charges

- Immediate publication on acceptance

- Inclusion in PubMed, CAS, Scopus and Google Scholar

- Research which is freely available for redistribution

Submit your manuscript at www.biomedcentral.com/submit 